\title{
Light reflection from nonlinear optical dielectric film on a bigyrotropic magnetoelectric substrate at angles close to Brewster angles
}

\author{
Yu. S. Dadoenkova and I. L. LyubchanskiI ${ }^{a}$
}

A. A. Galkin Donetsk Physicotechnical Institute of the National Academy of Sciences of Ukraine, ul. R. Lyuksemburg 72, Donetsk 83114, Ukraine

Department of Physics, Donetsk National University, ul. Universitetskaya 24, Donetsk 83005, Ukraine

Y. P. Lee

Quantum Photonic Science Research Center (q-Psi) and Department of Physics, Hanyang University, Seoul 133-791, Korea

\section{Th. Rasing}

Institute for Molecules and Materials, Radboud University Nijmegen 6525 ED, Nijmegen, The Netherlands (Submitted November 11, 2009; revised December 10, 2009)

Fiz. Nizk. Temp. 36, 673-679 (June 2010)

Light reflection from the interface of a dielectric film, characterized by a cubic optical nonlinearity, on a bigyrotropic substrate magnetoelectric substrate is investigated theoretically. Relations are obtained for the reflection coefficient as a function of the incidence angle of the light for the main magneto-optic configurations: polar, longitudinal, and transverse. The effect of the magneto-electric and magneto-optical contributions to the electric polarization of such a biaxial structure on the polarization state of the light reflected at angles close to the Brewster angles is studied. () 2010 American Institute of Physics. [doi:10.1063/1.3455805]

\section{INTRODUCTION}

The linear and nonlinear optical properties of magnetoelectric (ME) materials are being intensively studied theoretically and experimentally ${ }^{1,2}$ because of the unique properties of magnetoelectrics due to the direct interaction of the electric and magnetic subsystems, which determines the wide applications of ME structures in modern opto- and magnetoelectronics. Light reflection from uniform and structured ME media as well as from interfaces, which are characterized by ME properties, has been investigated in Refs. 1 and 3-9. Nonlinear optical phenomena due to the quadratic optical nonlinearity, such as second harmonic generation in a classic $\mathrm{ME}$ material $\mathrm{Cr}_{2} \mathrm{O}_{3}$, have been investigated theoretically ${ }^{10}$ and experimentally; ${ }^{11}$ see, also, the review
Ref. 12 where data on the nonlinear optics of magnetoelectrics are presented. However, the effect of a cubic nonlinear optical susceptibility (NOS) on the reflective power of the ME material has still not been studied. It should be expected that the cubic nonlinear optical interaction in combination with the linear ME interaction will result in changes in the refractive index of the nonlinear medium ${ }^{13}$ as well as in the reflection of light. These changes can be observed, for example, by studying light reflection at Brewster angles from the structure which is characterized by an ME tensor and a cubic NOS tensor. One realization of such a structure could be a dielectric film with large values of the cubic NOS on an NE substrate (MES). The case of a transverse magneto-optic configuration (MOC) with light reflection from such a struc-
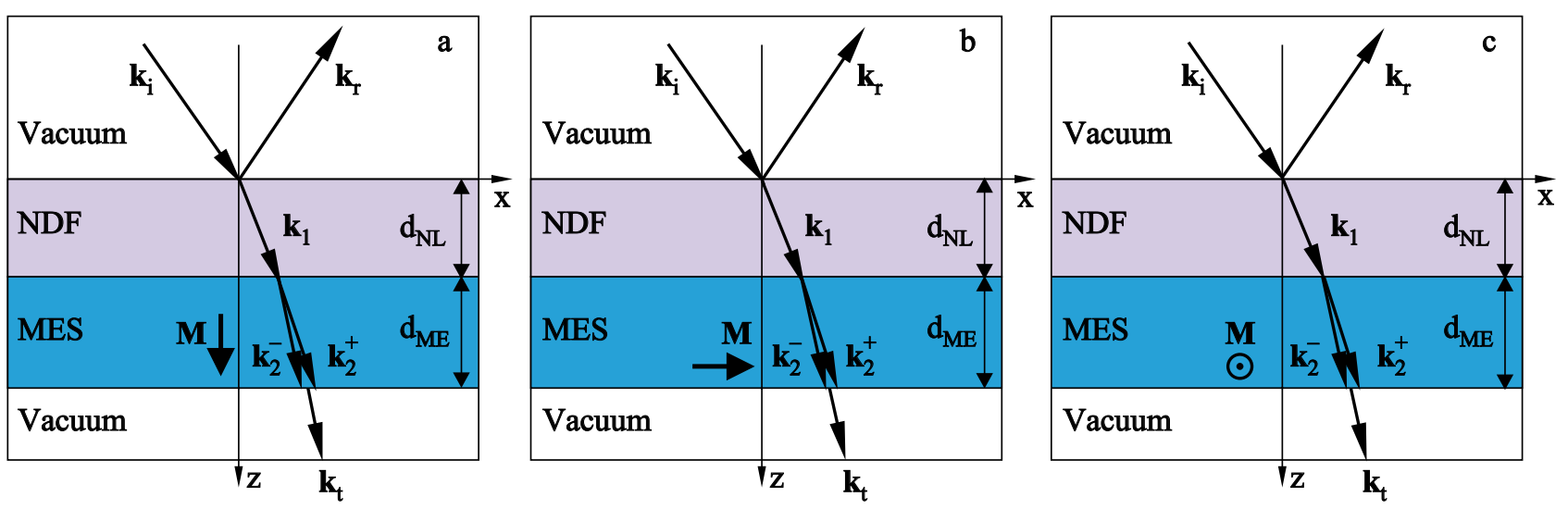

FIG. 1. Schematic diagram of the reflection of light from an NDF on a bigyrotropic MES in the following cases: polar MOC (a); longitudinal MOC (b); transverse MOC (c). $\mathbf{k}_{i}, \mathbf{k}_{r}$, and $\mathbf{k}_{t}$-wave vectors of incident, reflected, and transmitted waves in vacuum, respectively; $\mathbf{k}_{1}$-wave vector of the refracted wave in the NDF, $\mathbf{k}_{2}^{+}$and $\mathbf{k}_{2}^{-}$determine the wave vectors in the MES. 
ture was first investigated by us in Ref. 14, but we neglected the effect of the components of the ME tensor on the wave vectors of the normal electromagnetic waves (EMW) in an ME medium. In the present work the reflection of EMW from a dielectric film with cubic NOS on a substrate with ME interaction is studied theoretically for the main magnetooptic configurations (MOC): polar, longitudinal, and transverse. ${ }^{15,16}$ Glass doped with the semiconductor CdSe, which is characterized by large values of the components of the cubic $\mathrm{NOS}^{13}{ }^{13}$ is taken as the nonlinear dielectric film (NDF) and cubic yttrium-iron garnet (YIG) $\mathrm{Y}_{3} \mathrm{Fe}_{5} \mathrm{O}_{12}$ is taken as the MES. This substance is characterized in the near-IR by bigyrotropic properties, ${ }^{16}$ i.e. off-diagonal components, which depend strongly on the orientation of the magnetization vector $\mathbf{M}$ in $\mathrm{MES}$, are present in the dielectric and magnetic susceptibility tensors.

\section{PHENOMENOLOGICAL DESCRIPTION OF LIGHT REFLECTION FROM A BILAYER STRUCTURE}

We shall examine the reflection of a plane EMW from a bilayer structure consisting of a NDF which is characterized by a cubic optical nonlinearity and a MES with thicknesses $d_{N L}$ and $d_{M E}$ in the $X O Y$ plane; the $z$ axis is perpendicular to the surfaces of the NDF and the MES, as shown in Fig. 1. The main MOC are studied: polar $(\mathbf{M} \| O Z)$, longitudinal $(\mathbf{M} \| O X)$, and transverse $(\mathbf{M} \| O Z)$, as shown in Figs. 1a-1c, respectively.

The permittivity tensor $\varepsilon_{i j}$ and the magnetic susceptibility tensor $\mu_{i j}$ our system (see Fig. 1) can be represented in the following form:

$$
\begin{gathered}
\varepsilon_{i j}= \begin{cases}\delta_{i j}, & z<0, \\
\varepsilon_{i j}^{(1)}, & 0<z<d_{N L}, \\
\varepsilon_{i j}^{(2)}, & d_{N L}<z<d_{M E}, \\
\delta_{i j}, & z>d_{M E} .\end{cases} \\
\mu_{i j}= \begin{cases}\delta_{i j}, & z<0, \\
\delta_{i j}, & 0<z<d_{N L}, \\
\mu_{i j}^{(2)}, & d_{N L}<z<d_{M E}, \\
\delta_{i j}, & z>d_{M E} .\end{cases}
\end{gathered}
$$

The superscripts (1) and (2) denote the NDF and MES, respectively; $\delta_{i j}$ is the Kronecker delta function. The tensor $\varepsilon_{i j}^{(1)}$ has the following structure: $\varepsilon_{i j}^{(1)}=\varepsilon_{i j}^{(1 ; 0)}+\varepsilon_{i j}^{(1 ; N L)}$, where $\varepsilon_{i j}^{(1 ; 6)}$ and $\varepsilon_{i j}^{(1 ; N L)}=\chi_{i j k l}^{(3)} E_{k} E_{l}$ determine the linear and nonlinear parts of permittivity tensor and $\chi_{i j k l}^{(3)}$ is the third-order NOS tensor. Here $\varepsilon_{i j}^{(1 ; 0)}=\varepsilon_{i j}^{(1 ; 0)} \delta_{i j}$, and the tensors $\varepsilon_{i j}^{(2)}$ and $\mu_{i j}^{(2)}$ together with the diagonal tensors are characterized by the offdiagonal components, linear in $\mathbf{M}$, for each MOC (see the relations (10), (14), and (17)).

The propagation of an EMW in a NDF is described by the wave equation ${ }^{13}$

$$
\nabla \operatorname{div} \mathbf{E}-\Delta \mathbf{E}-k_{0}^{2} \hat{\varepsilon} \mathbf{E}=4 \pi \mathbf{P}^{N L(3)},
$$

where the cubic nonlinear polarization $\mathbf{P}^{N L(3)}$ at the incidentlight frequency $\omega$ is determined in the well-known manner: ${ }^{13}$

$$
P_{i}^{N L(3)}=\chi_{i j k l}^{(3)} E_{j} E_{k}^{*} E_{l} .
$$

In a medium with cubic symmetry $\mathbf{P}^{N L(3)}$ in the simplest case has the form

$$
\mathbf{P}^{N L(3)}=3 k_{0}^{2}\left(2 \chi_{1122}+\chi_{1221}\right) \mathbf{E}|\mathbf{E}|^{2} .
$$

Here $\chi_{1122}$ and $\chi_{1221}$ are the nonlinear components of the cubic NOS tensor. ${ }^{13}$

In the first, with respect to the components $\mathbf{P}^{N L(3)}$, approximation the solution of Eq. (2) can be represented in the form

$$
E_{i}(z)=E_{i}^{(0)}+4 \pi k_{0}^{2} \int_{0}^{d_{N L}} d z^{\prime} G_{i m}^{(1)}\left(z-z^{\prime}\right) P_{m}^{N L(3)}\left(z^{\prime}\right),
$$

where $E_{i}^{(0)}$ is the solution of the homogeneous wave equation. In the expression (5) the Green's functions $G_{i m}^{(1)}\left(z-z^{\prime}\right)$ are determined by the following equations:

$$
\hat{L}_{i m}\left(\partial_{z}\right) G_{m l}^{(1)}\left(z-z^{\prime}\right)=\delta_{i l} \delta\left(z-z^{\prime}\right)
$$

where

$$
\hat{L}_{i k}=\left(\begin{array}{ccc}
-k_{0}^{2} \varepsilon_{0}^{(1)}-\partial_{z}^{2} & 0 & i k_{x} \partial_{x} \\
0 & k_{x}^{2}-k_{0}^{2} \varepsilon_{0}^{(1)}-\partial_{z}^{2} & 0 \\
i k_{x} \partial_{x} & 0 & k_{x}^{2}-k_{0}^{2} \varepsilon_{0}^{(1)}
\end{array}\right),
$$

and $\partial_{x} \equiv \partial / \partial_{x}, \partial_{z}^{2} \equiv \partial^{2} / \partial z^{2}$.

In the MES the coupling of the electric field vector $\mathbf{E}$ and electric induction vector $\mathbf{D}$ as well as the coupling of the magnetic field vector $\mathbf{H}$ and the magnetic induction vector $\mathbf{B}$ give the material relations of the medium ${ }^{1}$

$$
D_{i}=\varepsilon_{i j}^{(2)} E_{j}+\alpha_{i j} H_{j}, \quad B_{i}=\mu_{i j}^{(2)} H_{j}+\alpha_{j i} E_{j},
$$

where $\hat{\varepsilon}^{(2)}$ and $\hat{\mu}^{(2)}$ are the permittivity and magnetic susceptibility tensors, respectively, and $\hat{\alpha}$ is the ME tensor which is diagonal in a cubic crystal $^{17}$

$$
\alpha_{i j}=\alpha \delta_{i j} .
$$

In the case of polar MOC the tensors $\varepsilon_{i j}^{(2)}$ and $\mu_{i j}^{(2)}$ of the MES have the following form:

$$
\varepsilon_{i j}^{(2)}=\left(\begin{array}{ccc}
\varepsilon^{(2)} & i \varepsilon^{\prime} & 0 \\
-i \varepsilon^{\prime} & \varepsilon^{(2)} & 0 \\
0 & 0 & \varepsilon^{(2)}
\end{array}\right), \quad \mu_{i j}^{(2)}=\left(\begin{array}{ccc}
\mu^{(2)} & i \mu^{\prime} & 0 \\
-i \mu^{\prime} & \mu^{(2)} & 0 \\
0 & 0 & \mu^{(2)}
\end{array}\right) \text {, }
$$

where $\varepsilon^{\prime}$ and $\mu^{\prime}$ are off-diagonal components, linear in the magnetization, of the permittivity and magnetic susceptibility tensors, respectively. In the explicit form of the tensors $\varepsilon_{i j}^{(2)}$ and $\mu_{i j}^{(2)}$ for each MOS we do not take account of the difference arising in the diagonal components of these tensors as a result of the quadratic magneto-optic interaction, which is important when studying magnetic birefringence. ${ }^{16}$ We shall take account of only the effect of the linear MO and $\mathrm{ME}$ interactions on light reflection, i.e. we assume that the corresponding diagonal components of the material tensors in Eqs. (10), (14), and (17) are equal.

We seek the solution of Maxwell's equations in the form of plane EMW: $\quad \mathbf{E}=\mathbf{E}_{0} \quad \exp [i(\mathbf{k} \cdot \mathbf{r}-\omega t)]$ and $\quad \mathbf{H}=\mathbf{H}_{0}$ $\exp [i(\mathbf{k} \cdot \mathbf{r}-\omega t)]$, where $\mathbf{E}_{0}$ and $\mathbf{H}_{0}$ are the amplitudes of the electric and magnetic fields of the EMW, respectively, $\mathbf{k}$ is 
the wave vector and $\omega$ is the frequency of the EMW. The system of Maxwell's equations in the MES can be written as follows:

$$
\left(\begin{array}{cccccc}
-\alpha & -s_{z} & 0 & -\mu^{(2)} & -i \mu^{\prime} & 0 \\
s_{z} & -\alpha & -s_{x} & i \mu^{\prime} & -\mu^{(2)} & 0 \\
0 & s_{x} & -\alpha & 0 & 0 & -\mu^{(2)} \\
\varepsilon^{(2)} & i \varepsilon^{\prime} & 0 & \alpha & -s_{z} & 0 \\
-i \varepsilon^{\prime} & \varepsilon^{(2)} & 0 & s_{z} & \alpha & -s_{x} \\
0 & 0 & \varepsilon^{(2)} & 0 & s_{x} & \alpha
\end{array}\right)\left(\begin{array}{c}
E_{x} \\
E_{y} \\
E_{z} \\
H_{x} \\
H_{y} \\
H_{z}
\end{array}\right)=0
$$

where $s_{x}$ and $s_{z}$ are the components of the dimensionless wave vector $\mathbf{s}=\mathbf{k} / k_{0}, k_{0}=\omega / c$, and $c$ is the speed of light in vacuum.

The homogeneous system of algebraic equations (11) possesses a nontrivial solution if its determinant is zero. Satisfying this condition, we obtain a quartic algebraic equation for the components of the dimensionless wave vector $s_{x}$ and $s_{z}$. Assuming $s_{x}$ to be an independence variable and solving this equation for $s_{z}$ we obtain

$$
\left(s_{z}^{ \pm(p)}\right)^{2}=\frac{-B^{(p)} \pm \sqrt{D^{(p)}}}{2 A}, \quad D^{(p)}=\left(B^{(p)}\right)^{2}-4 A C^{(p)},
$$

where

$$
\begin{aligned}
A= & \varepsilon^{(2)} \mu^{(2)}-\alpha^{2}, \\
B^{(p)}= & 2 A s_{x}^{2}+2 \alpha^{2}(b+\varepsilon \mu)-\Delta \varepsilon \Delta \mu 2 \alpha^{4} \\
C^{(p)}= & A s_{z}^{4}+s_{x}^{2}\left(-2 \alpha^{4}+2 \alpha^{2}(b+\varepsilon \mu)-2 \varepsilon \mu b\right)+\varepsilon \mu \Delta \varepsilon \Delta \mu \\
& -\alpha^{2}(\Delta \varepsilon \Delta \mu+2 \varepsilon \mu b)+\alpha^{4}(2 b+\varepsilon \mu)-\alpha^{6}, \\
b= & \varepsilon^{(2)} \mu^{(2)}+\varepsilon^{\prime} \mu^{\prime}, \quad f=\left(\varepsilon^{(2)}\right)^{2} \Delta \mu+\left(\mu^{(2)}\right)^{2} \Delta \varepsilon \\
\Delta \varepsilon= & \left(\varepsilon^{(2)}\right)^{2}-\left(\varepsilon^{\prime}\right)^{2}, \quad \Delta \mu=\left(\mu^{(2)}\right)^{2}-\left(\mu^{\prime}\right)^{2} .
\end{aligned}
$$

For longitudinal MOC the tensors $\varepsilon_{i j}^{(2)}$ and $\mu_{i j}^{(2)}$ for the MES have the form

$$
\varepsilon_{i j}^{(2)}=\left(\begin{array}{ccc}
\varepsilon^{(2)} & 0 & 0 \\
0 & \varepsilon^{(2)} & i \varepsilon^{\prime} \\
0 & -i \varepsilon^{\prime} & \varepsilon^{(2)}
\end{array}\right), \quad \mu_{i j}^{(2)}=\left(\begin{array}{ccc}
\mu^{(2)} & 0 & 0 \\
0 & \mu^{(2)} & i \mu^{\prime} \\
0 & -i \mu^{\prime} & \mu^{(2)}
\end{array}\right) .
$$

Calculations similar to those presented above for a polar MOC make it possible to find the dimensionless wave vector $s_{z}^{(l)}$, which has the form

$$
\left(s_{z}^{ \pm(l)}\right)^{2}=\frac{-B^{(l)} \pm \sqrt{D^{(l)}}}{2 A}, \quad D^{(l)}=\left(B^{(l)}\right)^{2}-4 A C^{(l)},
$$

where

$$
\begin{aligned}
B^{(l)}= & 2 A s_{x}^{2}-2 \varepsilon \mu b+2 \alpha^{2}(b+\varepsilon \mu)-2 \alpha^{4} \\
C^{(l)}= & A s_{z}^{4}+s_{x}^{2}\left(-2 \alpha^{4}+2 \alpha^{2}(b+\varepsilon \mu)-f\right) \\
& +\varepsilon \mu \Delta \varepsilon \Delta \mu-\alpha^{2}(\Delta \varepsilon \Delta \mu-2 \varepsilon \mu b)+\alpha^{4}(2 b+\varepsilon \mu) \\
& +\varepsilon \mu \Delta \varepsilon \Delta \mu-\alpha^{6}
\end{aligned}
$$

For a transverse MOC the tensors $\varepsilon_{i j}^{(2)}$ and $\mu_{i j}^{(2)}$ for MES have the form

$$
\varepsilon_{i j}^{(2)}=\left(\begin{array}{ccc}
\varepsilon^{(2)} & 0 & i \varepsilon^{\prime} \\
0 & \varepsilon^{(2)} & 0 \\
-i \varepsilon^{\prime} & 0 & \varepsilon^{(2)}
\end{array}\right), \quad \mu_{i j}^{(2)}=\left(\begin{array}{ccc}
\mu^{(2)} & 0 & i \mu^{\prime} \\
0 & \mu^{(2)} & 0 \\
-i \mu^{\prime} & 0 & \mu^{(2)}
\end{array}\right) .
$$

Performing calculations similar to those just described we obtain the wave vectors $s_{z}^{(l)}$ in the MES:

$$
\left(s_{z}^{ \pm(t)}\right)^{2}=\frac{-B^{(t)} \pm \sqrt{D^{(t)}}}{2 A}, \quad D^{(t)}=\left(B^{(t)}\right)^{2}-4 A C^{(t)},
$$

where

$$
\begin{aligned}
B^{(t)}= & 2 A^{(t)} s_{x}^{2}-f+2 b \alpha^{2}-2 \alpha^{4}, \\
C^{(t)}= & A^{(t)} s_{x}^{4}+s_{x}^{2}\left[-f+2 b \alpha^{2}-2 \alpha^{4}\right]+\varepsilon^{(2)} \mu^{(2)} \Delta \varepsilon \Delta \mu \\
& -\alpha^{2}\left[2 \varepsilon^{(2)} \mu^{(2)} b+\Delta \varepsilon \Delta \mu\right]+\alpha^{4}\left(2 b+\varepsilon^{(2)} \mu^{(2)}\right)-\alpha^{6} .
\end{aligned}
$$

The amplitudes of the reflected-light fields $\mathbf{E}^{(r)}$ can be expressed in terms of the amplitudes of the incident-light fields $\mathbf{E}^{(i)}$ by means of the matrix $\hat{R}$ of reflection coefficients:

$$
\left(\begin{array}{c}
E_{s}^{(r)} \\
E_{p}^{(r)}
\end{array}\right)=\hat{R}\left(\begin{array}{c}
E_{x}^{(i)} \\
E_{p}^{(i)}
\end{array}\right)
$$

where the subscripts $p$ and $s$ denote the $p$ and $s$ polarized EMW, respectively.

The matrix $\hat{R}$ of the reflection coefficients for the present system can be represented in the following form:

$$
\hat{R}=\left(\begin{array}{ll}
R_{s s} & R_{p s} \\
R_{s p} & R_{p p}
\end{array}\right), \quad R_{i j}=R_{i j}^{(0)}+R_{i j}^{(1)} .
$$

Here $R_{i j}^{(0)}(i, j=s, p)$ are the components of the reflection matrix in the zeroth approximation, i.e. neglecting the nonlinear polarization (2), $R_{i j}^{(1)}$ corresponds to the first-approximation corrections to $\hat{R}$. We note that the off-diagonal components of $R_{s p}$ and $R_{p s}$ of the reflection matrix (21) appear because of bigyrotropy and the ME interaction in the substrate.

\section{RESULTS AND ANALYSIS}

As mentioned in the introduction, we shall examine a bilayer structure consisting of a silicon dioxide film, doped with cadmium selenide, and a YIG substrate in which the linear ME effect is observed near crystal defects ${ }^{18}$ and at low temperatures after the sample is cooled in simultaneously applied electric and magnetic fields. ${ }^{19}$ It is noted in Ref. 3 that the values of the ME constants in epitaxial YIG films can reach the maximum value $\alpha \sim 10^{-2}$ at room temperature.

The following values of the material parameters were used in the calculations: $\varepsilon^{(1 ; 0)}=4, \quad \chi^{(3)} \sim 10^{-9}$ esu, $\varepsilon^{(2)}$ $=4.5796, \mu^{(2)}=1, \mu^{\prime}=8.76 \cdot 10^{-5}, \varepsilon^{\prime}=-2.47 \cdot 10^{-4}, \alpha=10^{-3}$ for wavelength $\lambda=1.15 \mu \mathrm{m}$. The amplitude of the electric field of the incident EMW is $E \sim 10^{8} \mathrm{~V} / \mathrm{m}$, and the MES thickness is $d_{N L}=\lambda, 3 \lambda, 5 \lambda$. We note that the assumption 

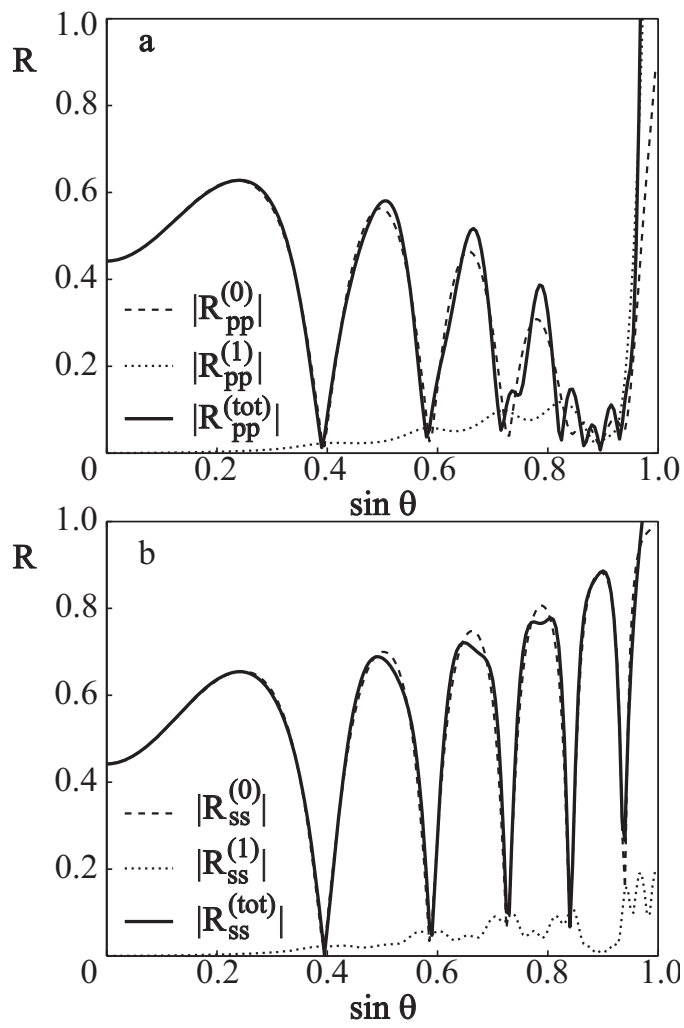

FIG. 2. Curves of the moduli of the diagonal reflection coefficients of a bilayer structure with $d_{N L}=\lambda, d_{M E}=10 \lambda$ versus $\sin \theta$. The dashed lines correspond to the reflection coefficients neglecting the nonlinearity of the film $\left|R_{i i}^{(0)}\right|$, the dotted lines show the first-approximation corrections $\left|R_{i i}^{(1)}\right|$ to the reflection coefficients, and the solid lines show the resulting reflection coefficients $\left|R_{i i}^{(0)}+R_{i i}^{(1)}\right|=\left|R_{i i}^{\text {tot }}\right|, i=s, p$ for a polar MOC in the cases $s$-polarized EMW $\left(\left|R_{s s}\right|\right)$ (a) and $p$-polarized EMW $\left(\left|R_{p p}\right|\right)(\mathrm{b})$.
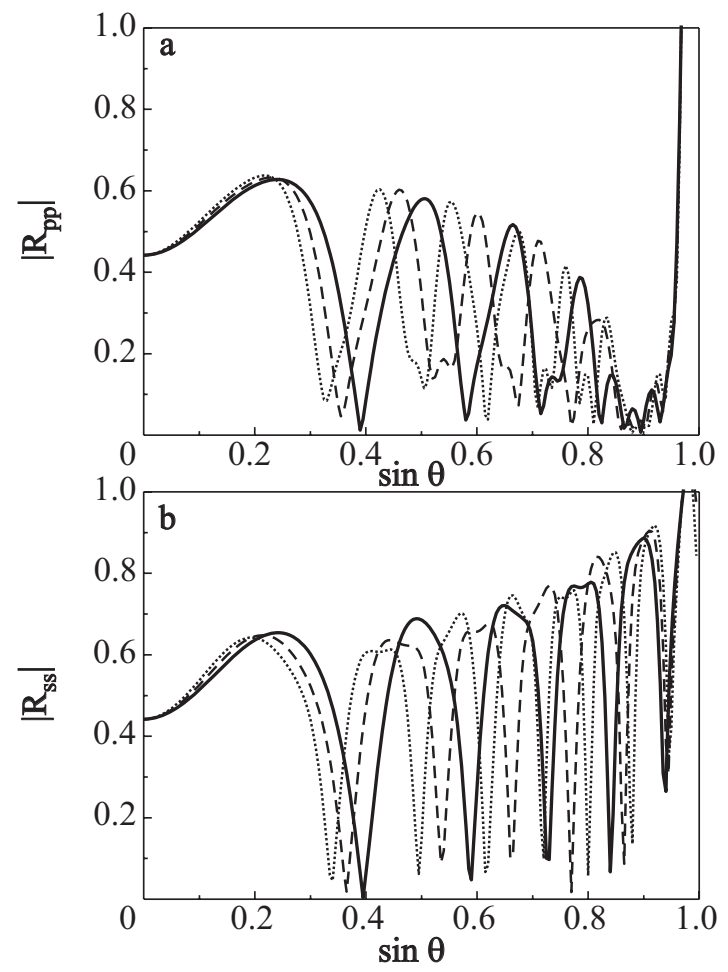

FIG. 3. Moduli of the diagonal reflection coefficients $R_{p p}$ (a) and $R_{s s}$ (b) versus $\sin \theta$ for different thicknesses of the nonlinear film: $d_{N L}=\lambda$ (solid lines), $d_{N L}=3 \lambda$ (dashed lines), and $d_{N L}=5 \lambda$ (dotted lines).
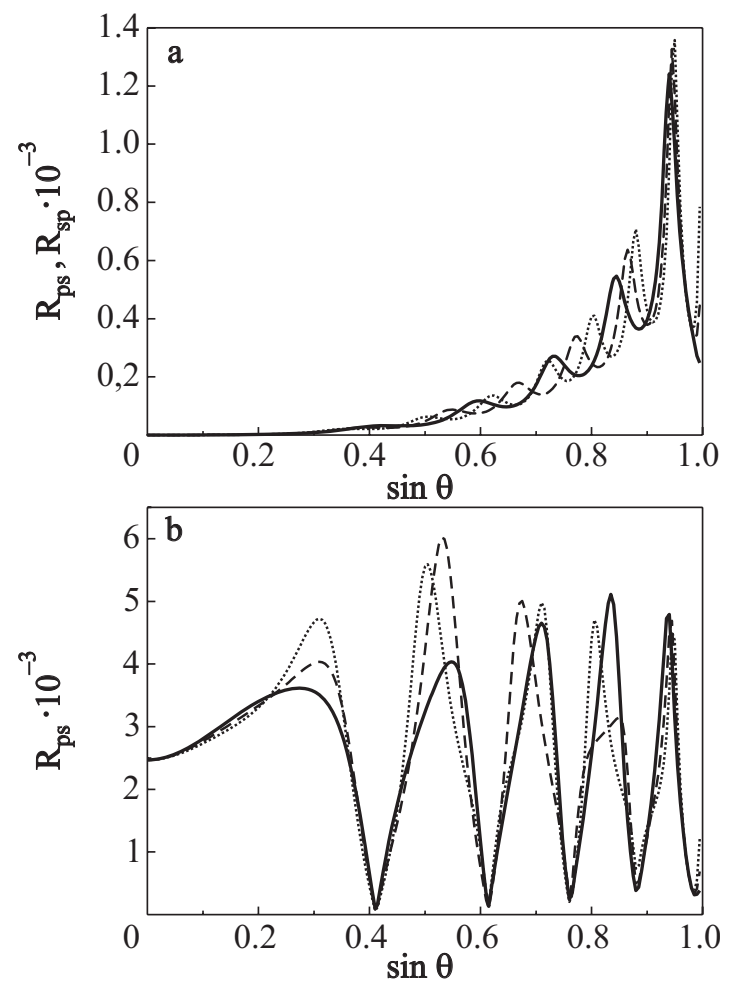

FIG. 4. Off-diagonal reflection coefficients $\left|R_{p s}\right|$ versus $\sin \theta$ for different values of the thickness of the nonlinear film: $d_{N L}=\lambda$ (solid lines), $d_{N L}=3 \lambda$ (dashed lines), and $d_{N L}=5 \lambda$ (dotted lines) for polar and longitudinal MOC in cases of negligibly small ME interaction in MES $\alpha \rightarrow 0$ (a) and $\alpha=10^{-2}$ (b).

made above that the diagonal components of the permittivity and permeability tensors are equal is applicable to a YIG film.

Figure 2 displays plots of the moduli of the amplitudes of the reflection coefficients neglecting the optical nonlinearity of the film $\left|R_{i i}^{(0)}\right|$, corrections to the first Born approximation $\left|R_{i i}^{(1)}\right|$, and the resulting reflection coefficients $\mid R_{i i}^{(0)}$ $+R_{i i}^{(1)}|=| R_{i i}^{\mathrm{tot}} \mid(i=s, p)$ versus the sine of the reflection angle $\sin \theta$ (dashed, dotted, and solid lines, respectively) in the case of polar MOC. It is evident in Fig. 2 that the contribution of the cubic nonlinearity to the reflection coefficient reaches $5 \%$.

Figures 3-5 show the moduli of the amplitude coefficients of reflection of the main components $R_{s s}$ and $R_{p p}$ and the cross-polarized components $R_{s p}$ and $R_{p s}$ of the EMW as functions of $\sin \theta$ for different thicknesses of the NDF $d_{N L}$ $=\lambda$ (solid lines), $d_{N L}=3 \lambda$ (dashed lines), and $d_{N L}=5 \lambda$ (dotted lines).

Figures $3 \mathrm{a}$ and $3 \mathrm{~b}$ show the diagonal components of the reflection matrix as functions of $\sin \theta$. For the main MOC the diagonal reflection coefficients $R_{p p}$ and $R_{s s}$ depend on the ME interaction constant $\alpha$ very weakly. The difference between $\left.R_{i i}\right|_{\alpha \rightarrow 0}$ and $\left.R_{i i}\right|_{\alpha=10^{-2}}(i=s, p)$, does not exceed a hundredth of a percent. Moreover, $R_{p p}$ and $R_{s s}$ for the MOC considered are equal to one another with within thousandths of a percent.

Figures $4 \mathrm{a}$ and $5 \mathrm{a}$ show plots of the off-diagonal components $\left|R_{p s}\right|$ and $\left|R_{s p}\right|$ of the reflection matrix versus $\sin \theta$ with an infinitesimal ME interaction in the MES $(\alpha \rightarrow 0)$ for 

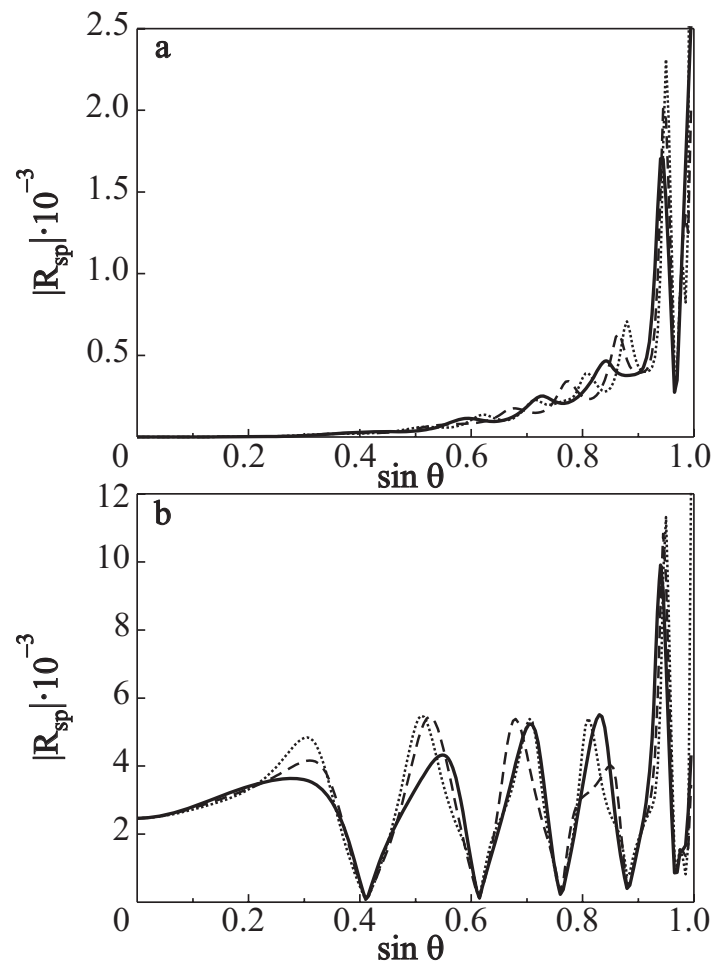

FIG. 5. Off-diagonal reflection coefficients $\left|R_{s p}\right|$ versus $\sin \theta$ for different values of the thickness of the nonlinear film: $d_{N L}=\lambda$ (solid lines), $d_{N L}=3 \lambda$ (dashed lines), and $d_{N L}=5 \lambda$ (dotted lines) for polar and longitudinal MOC in cases of negligibly small ME interaction in MES $\alpha \rightarrow 0$ (a) and $\alpha=10^{-2}$ (b).

different thicknesses of the NDF $d_{N L}$ in the case where the magnetization vector lies in the incidence plane, i.e. for polar and longitudinal MOC. For transverse MOC, as $\alpha \rightarrow 0$ normal EMW in the medium transform into independence TE and TM modes, and the reflection matrix becomes diagonal. When the ME interactions is taken into account, instead of the linearly polarized TE and TM modes the normal EMW in the medium will be waves with elliptical (right- and lefthand) polarizations. Therefore, when the magnetization vector is perpendicular to the incidence plane, the ME interaction will lead to the appearance of cross-polarized components $R_{p s}$ and $R_{s p}$ in the reflection matrix. Figures $4 \mathrm{~b}$ and $5 \mathrm{~b}$ illustrate the analogous dependences $\left|R_{p s}\right|$ and $\left|R_{s p}\right|$ with $\alpha=10^{-2}$ for the main MOC. It is evident in Figs. 3 and 4 that the off-diagonal components of the reflection matrix are three or four orders of magnitude smaller in magnitude than the diagonal components. Comparing Figs. $4 \mathrm{a}$ and $4 \mathrm{~b}$ and Figs. 5a and 5b shows that the off-diagonal components depend strongly on the ME interaction, which results in higher values of the off-diagonal coefficients of reflection at angles of incidence far from glancing incidence.

It is evident in Figs. 3-5 that the plots of the diagonal and off-diagonal reflection coefficients have several minima, which do not reach zero. Therefore, when light is reflected from the two-layer system under study it is impossible to obtain completely polarized, reflected light. In this case the minima of the reflection coefficients correspond to reflection at so-called pseudo-Brewster angles (PBA).

Figures $6 \mathrm{a}$ and $6 \mathrm{~b}$ show two-dimensional plots of the evolution of the diagonal coefficients of reflection $\left|R_{p p}\right|$ and $\left|R_{s s}\right|$ as a function of the NDF thickness, whence one can see that the minima of the reflection coefficients tend to shift in

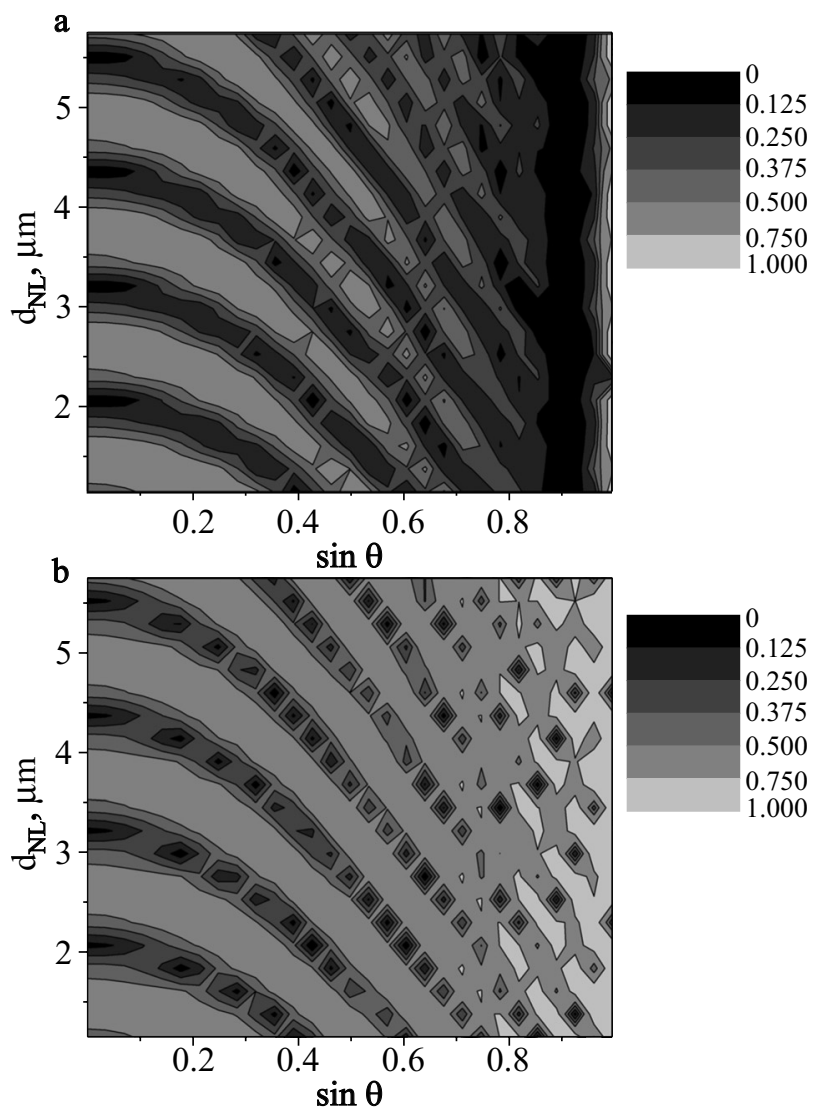

FIG. 6. Two-dimensional plot of the evolution of the diagonal reflection coefficients as functions of $\sin \theta$ and the thickness of the nonlinear film $d_{N L}$ in the cases of all main MOC: $\left|R_{p p}\right|$ (a) and $\left|R_{s s}\right|$ (b).

the direction of smaller angles of incidence $\theta$ with increasing thickness $d_{N L}$ of the nonlinear film for both $s$ - and $p$-polarized reflected waves. This results in a similar shift of the PBA.

Figure 7 shows a two-dimensional plot of the evolution of the off-diagonal reflection coefficient $\left|R_{p s}\right|$ with increasing

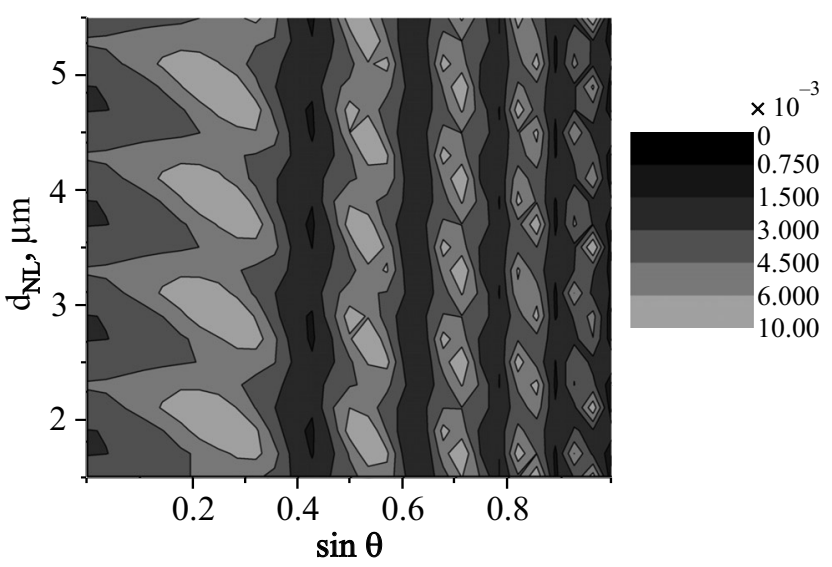

FIG. 7. Two-dimensional plot of the evolution of the off-diagonal reflection coefficient $\left|R_{p s}\right|$ as a function of $\sin \theta$ and the thickness of the nonlinear film $d_{N L}$ in the case of a polar MOC. 
thickness of the nonlinear film in the case $\alpha=10^{-2}$ for polar MOC. The off-diagonal components of the reflection matrix for the main MOC have the same form, shown in Fig. 7.

\section{CONCLUSION}

The reflection coefficients of a bilayer medium consisting of a nonlinear optical film with a cubic optical nonlinearity on a magneto-electric bigyrotropic substrate were obtained. The contribution of the cubic optical nonlinearity to the polarization state of the reflected light was investigated theoretically. The effect of the ME properties of the substrate and the cubic optical nonlinearity of the film on the diagonal and off-diagonal components of the reflection matrix was investigated for media with cubic symmetry (YIG and glass doped with the semiconductor $\mathrm{CdSe}$ ), forming a bilayer structure. The calculations showed that even when the ME tensor is characterized by a single nonzero component the effect of the ME interaction on the reflection of polarized light will be substantial. It was shown that the bigyrotropic and ME properties of the substrate make it impossible to obtain completely polarized reflected light, so that reflection under Brewster's angle is impossible. However, reflection occurs at so-called pseudo-Brewster angles, where the diagonal components of the reflection matrix reach minimal (but nonzero) values. We also note that for transverse MOC, offdiagonal components appear in the reflection matrix because of the ME interaction. As the thickness of the nonlinear film increases, the PBA shifts appear and the minimal values of the reflection coefficients increase.

This work was supported by the grant FP7-People-2009IRSES, No. 247556 (Yu. S. D. and I. L. L., Ukraine), Grants NRF (Y. P. L., Korea), and grants NWO and NanoNed (T. R., Netherlands).

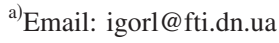

${ }^{1}$ T. H. O'Dell, The Electrodynamics of Magnetoelectric Media, NorthHolland, Amsterdam (1970).

${ }^{2}$ M. Fiebig, J. Phys. D 38, R123 (2005).

${ }^{3}$ R. V. Pisarev, Ferroelectrics 162, 191 (1994).

${ }^{4}$ B. B. Krichevtsov, V. V. Pavlov, R. V. Pisarev, and V. N. Gridnev, J. Phys.: Condens. Matter 5, 8233 (1993).

${ }^{5}$ I. E. Chupis, Ferroelectrics 204, 173 (1997).

${ }^{6}$ E. B. Graham and R. Raab, J. Phys.: Condens. Matter 9, 1863 (1997).

${ }^{7}$ V. N. Gridnev, Phys. Rev. B 51, 13079 (1995).

${ }^{8}$ N. Kida, Y. Kaneko, I. P. He, N. Matsubara, H. Sato, T. Arima, H. Akoh, and Y. Tokura, Phys. Rev. Lett. 96, 167202 (2006).

${ }^{9}$ H. Yamada, H. Sato, H. Akoh, N. Kida, T. Arima, M. Kawasaki, and Y. Tokura, Appl. Phys. Lett. 92, 062508 (2008).

${ }^{10}$ S. B. Borisov, N. N. Dadoenkova, I. L. Lyubchanskiı̌, and V. L. Sobolev, Fiz. Tverd. Tela (Leningrad) 32, 3668 (1990) [Sov. Phys. Solid State 32, 2127 (1990)].

${ }^{11}$ M. Fiebig, D. Frohlich, B. B. Krichevtsov, and R. V. Pisarev, Phys. Rev. Lett. 73, 2127 (1994).

${ }^{12}$ M. Fiebig, V. V. Pavlov, and R. V. Pisarev, J. Opt. Soc. Am. B 22, 96 (2005).

${ }^{13}$ R. W. Boyd, Nonlinear Optics, Academic Press, San Diego (1992).

${ }^{14}$ Yu. S. Dadoenkova, I. L. Lyubchanskii, Y. P. Lee, and Th. Rasing, Eur. Phys. J. B 71, 401 (2009).

${ }^{15}$ G. A. Smolenskiı̌ and V. V. Lemanov, Ferrites and Their Technical Applications, Nauka, Leningrad (1975).

${ }^{16}$ G. S. Krinchik, Physics of Magnetic Phenomena, Moscow State University Press, Moscow (1976).

${ }^{17}$ R. R. Birss, Symmetry and Magnetism, North-Holland, Amsterdam (1966).

${ }^{18}$ R. V. Pisarev, B. B. Krichevtsov, V. V. Pavlov, and A. G. Selitsky, J. Magn. Soc. Jpn. 11, 33 (1987).

${ }^{19}$ H. Ogawa, E. Kita, Y. Mochida, K. Kohn, S. Kimura, A. Tasaki, and K. Siratori, J. Phys. Soc. Jpn. 56, 452 (1987).

Translated by M. E. Alferieff 